\title{
Assessment of Antiretroviral Therapy Outcomes among Key Populations after 6 Months of Initiation in Nigeria
}

\author{
Article by Ugbena, Eneojo Richard ${ }^{1}$, Iwuagwu, Stella ${ }^{2}$, Okekearu, Ifeanyi ${ }^{3}$, Wole, \\ Fajemisin ${ }^{4}$, Ioytim Isa ${ }^{5}$ \\ ${ }^{1}$ Society for Family Health, Nigeria \\ ${ }^{2}$ Centre for the right to Health \\ ${ }^{3}$ Society for Family Health, Nigeria \\ ${ }^{4}$ Society for Family Health, Nigeria \\ ${ }^{5}$ USAID, Nigeria \\ E-mail: rugbena@yahoo.com ${ }^{1}$
}

\begin{abstract}
Introduction: Key Populations are disproportionately affected by HIV/AIDS in all settings. Unfortunately, reaching KPs with HIV intervention is a huge challenge because of stigma, discrimination, violence, and criminalization of KPs lifestyles. Because of lack of KP specific interventions, data on KPs ART outcomes is lacking. This study determined ART outcomes among KPs 6-months after initiation.

Methodology: The study was a longitudinal study of all HIV positive KPs initiated on ART in 7 One-Stop-Shops from October 2016-March 2017. Both Time-1 and Time-2 data were abstracted from facility record after initiation and 6-month after for each patient. Data abstracted include: age, sex, marital status, educational level, employment status and ART outcome 6 months after initiation.

Results: Sixty-seven percent of patients were retained on ART, $1.8 \%$ died, $20.6 \%$ were lost to follow up, $4.5 \%$ transferred to other facilities and 6.2\% stopped ART by the end of the $6^{\text {th }}$ month. PWID had highest retention rate (74.6\%) more than MSM (70.2\%), while FSW had the least retention rate $(65.4 \%)$ but highest transfer out rate (4.9\%) more than MSM (4.7\%). Factors positively associated with retention at $6^{\text {th }}$ months include: Being a male $P=0.007$; higher education $P=0.000$; employed patient $P=0.000$; living with sex partner $P=0.000$.

Conclusion: This study has laid a baseline data for ART outcomes among KPs in Nigeria. Further study is however required to identify factors that could improve retention on ART among KPs. This is important if we must achieve the second and third 90s of UNAIDs 90.90 .90 goals.
\end{abstract}

Keywords: HIV/AIDS, Antiretroviral therapy; Key Population in Nigeria; One-Stop-Shop; Antiretroviral therapy outcomes.

\section{Introduction}

HIV/AIDS has remained a significant public health concern for over three decades and even though a lot of studies were carried out among the general population, data is less available for KPs owning to challenges associated with reaching KPs with health services and data collection especially in Africa. Majority of the world's new HIV infections occur in resource poor-countries, [1] with twothirds of the world's HIV-infected population living in Africa [2] Nigeria has the second largest HIV epidemic in the world and has one of the highest new infection rates in sub-Saharan Africa [3]. However, in recent years there has been a remarkable achievement in combating global HIV with new infection declining in most part of the world except for Eastern Europe and Central Asia region where since 2010, the annual number of new infections in the region has climbed by an alarming $60 \%$ [4]. In 2016, there were roughly 1.8 million new HIV infections [1], a decline from 2.1 million new infections in 2015 [4].

In all countries and settings, KPs are disproportionately affected by HIV infection [5]. KPs are groups that have a high risk and disproportionate burden of HIV in all epidemic settings due to their behavioural patterns and specific legal and social challenges that increase their vulnerability to HIV, including barriers to accessing HIV prevention, treatment and other health and social services [6], [7]. 
Key populations include Men who have Sex with Men (MSM), People Who Inject Drugs (PWID), and Sex Workers (SW), people in prisons and closed settings, and transgender people [8]. UNAIDS estimates that between 40 and 50 percent of all new HIV infections among adults worldwide occur in these key populations and among their sex partners [5], [9]. In Nigeria, It was estimated that SWs, MSM, and PWID make up 3.4\% of Nigerian population, yet they account for $32 \%$ of new HIV infections [10]. The prevalence of HIV among MSM, brothel-based FSW, none brothel-based FSW and PWID in Nigeria was $23 \%, 19.4 \%, 8.6 \%$ and $3.4 \%$ respectively [11] compared to the national prevalence of 3.0 [12]. This clearly shows a disproportionately higher burden among KPs in Nigeria and they will continue to drive the epidemic. HIV/AIDS intervention among KPs remain a priority if we must achieve the ambitious UNAIDS 90.90 .90 goals by 2020 [9]. However, reaching KPs with HIV intervention is a huge challenge because of stigma, discrimination, violence and in some cases criminalization of KP behavioural patterns [5]. These factors have often limited KPs access to and utilization of comprehensive HIV prevention, treatment, care, and support services [13]. Even when services are available, HIV-related stigma within healthcare settings may act as a barrier to the uptake and delivery of HIV care [14]. In Nigeria, there is stigma and discrimination as well as legislation against MSM, PWID and SWs lifestyle. Punitive laws [3], against homosexuality have meant that men who have sex with men are now even more vulnerable to HIV infection and face many difficulties accessing HIV services.

After HIV diagnosis [15], timely entry into HIV medical care and retention in care are essential to the provision of effective antiretroviral therapy. ART programmatic success requires that patients who are taking ART remain on treatment and are followed up regularly to monitor ART service outcomes. Recent data from the U.S. Centres for Disease Control and Prevention [15] in the USA reveal that of those who knew they had HIV, only $69 \%$ were linked to care, and only 59\% were retained in care. Once patients are in care and are receiving treatment [15], high levels of adherence are required to prevent the selection of resistance mutations and subsequent virologic failure. From existing literature [16] adherence to ART was reported to be influenced by age, access to healthcare, the burden of multiple vulnerabilities, policy involving risk behaviours and mental health. In addition, programmatic factors such as quality of counselling, location of service point, health worker attitude may be some factors why some patients initiated on ART may not be retained or adhere to ART service guidelines. There are various notable event that occurs among patients on ART over time; the patient may adhere to treatment by keeping to clinic appointment and pill intake; others may be lost to follow up when they failed to show up in clinic 90 days after the last clinic visit and effort to trace and return them to services failed; some may die from the disease complication, some may stop ART completely for any reason or stopped at a point and then re-start again. These various outcomes are characteristic of a typical ART program. Some of long-term retrospective studies in Nigeria have described various ART outcomes among the general population. In a study of long-term outcomes on antiretroviral therapy carried out in multiple sites from 2008- 2012, [17] of the 70,002 patients, $1.8 \%$ were reported as having died, $30.1 \%$ were lost to follow-up, and $0.1 \%$ withdrew from treatment. Another retrospective cohort analysis of 3,496 patients initiated on ART from 35 sites across the country between 2004 to 2012 showed that $1.1 \%$ died, 12.3\% LTFU, 13.9\% stopped ART with the highest attrition in the first 3 months [18] However, a similar 10 years retrospective study conducted in a facility [19] showed that attrition is highest in the first year of initiation (Overall attrition rate was 1.52 per 1000 person-months (PM) with 2.68/1000 PM in the first year, 1.81/1000 PM in the first 5 years, and 0.75 in the last 5 years. Similar data among KPs is currently lacking in Nigeria. While the MSM population does better than other HIV infected KPs with regard to linkage to and retention in care, little is known about engagement in care outcomes for important subpopulations of MSM [19] like the homosexual or bisexual MSMs. In a national cohort of American veterans, being MSM was associated with improved adherence to a visit in each of the 4 quarters of a year [20]. For PWID, while retention was found to be less at early years of initiation and improved after some years [21] a study in Vietnam showed good retention and immunological response to ART among a predominantly PWID group of patients despite advanced HIV infections at baseline; $88.4 \%$ after 6 months to $74.6 \%$ at 36 months [22] Among FSW, retention was more among FSW who have positive perception of HIV service providers [23]. With expanded ART programs in resource-limited settings which have 
improved treatment access to millions of People Living with HIV (PLHIV), inadequate retention in care has been a real challenge for the programs and has undermined efforts to optimize patient and program outcomes [24]. This challenge on ART retention may be worst for KPs in Africa because of the challenges with accessing health services due to social stigma, gender-based violence, discriminatory policies including criminalization of the lifestyles of key populations, which has hindered their access to care and treatment. In addition, these groups are hard to reach with health interventions and behaviour change interventions because they are often mobile and "hidden." [25]

Because KPs are drivers of HIV epidemic, reaching members of these communities with evidencebased interventions that improve their access to and uptake of services across the HIV prevention, care, and treatment cascade is essential to achieving the UNAIDS 90-90-90 goals [5] Currently the government of Nigeria does not provide any KP specific health services thus, public health intervention for KPs is only provided through donor-supported projects. One of such project is the Strengthening HIV Prevention Services for Most at Risk Population (SHiPS for MARPS), a PEPFAR funded project through USAID Nigeria with focus on providing comprehensive prevention services including treatment as prevention for KPs within the communities. The project implemented minimum prevention package for KPs conducted HIV counselling and testing and established OneStop-Shops for comprehensive health services for KPs. The SHiPS for MARPS project was implemented by Society for Family Health Nigeria in consortium with Population Service International and Center for the Right to Health in 7 states. The community health services provided by SHiPS for MARPS project offers an opportunity for KPs who hitherto, had a poor attitude to HIV counselling and testing to know their HIV status. With increased uptake of HIV counselling and testing among KPs in the communities, more HIV positive KPs were identified and linked to treatment. The project implemented "test and treat" strategy in line with WHO guideline. This implies that all newly identified positive KPs were initiated same day or at most, within the next 2 weeks. This strategy increased the number of KPs initiated on treatment. However, retention of treatment after initiated may be an issue particularly for mobile and hidden population such as KPs. Couple with poor health seeking behaviours, stigma and discrimination, maintaining those already initiated on ART may be a serious challenge. It is therefore important to determine the various ART outcomes such as retention rate, stopped ART, lost to follow up, transferred-out and death among the subpopulations of KPs. These factors are important for an effective HIV intervention, particularly among the KPs. This study will determine what proportion of positive KPs linked to ART are retained on treatment 6 months after initiation, and for those not retained on treatment, what event happened.

\section{Objectives}

To evaluate ART outcomes among KPs attending OSS in the first 6-7 months after initiation

\section{Specific objectives}

- To determine the proportion of KPs who are retained on ART 6-7 months after initiation of ART.

- To determine the attrition rate (Loss to follow up, dead, stopped ART) in the first 6-7 months after initiation on ART.

- To determine the proportion of KPs who transferred to other facilities in the first 6-7 months of initiation on ART.

- To determine what proportion re-started ART in the first 6-7 months of initiation on ART.

\section{Methodology}

\section{Site}

This study was carried out across 7 states supported by SHiPS for MARPS project. In each of the state, the project established One-Stop-Shop facility to provide HIV prevention, treatment, and care services for KPs and their partners. Other services provided at the sites include cervical cancer screening, syndromic management of other sexually transmitted diseases, partner notification services. 


\section{Study design}

The study was a longitudinal study of all HIV positive KPs who were initiated on ART in 7 OSS from October 2016 to March 2017. After initiation, time 1 data were abstracted from medical record of each patient to obtain their baseline characteristics. Each patient was then followed up for at least six months when they are eligible for viral load testing according to national ART guideline. Thus, the last patient attained 6 months by September 2017. The second data abstraction was carried out after the $6^{\text {th }}$ month of initiation for each patient to determine ART outcome if they are still receiving ART services. The $6^{\text {th }}$-month visit for some patients fell within 6 to 8 months after initiation depending on the clinic appointment.

\section{Sampling technique}

All patients initiated on ART between October 2016 and March 2017 in the 7 OSS were eligible for the study and data was abstracted for all of them. But all patients who were transferred to the facilities during the enrolment period were excluded to avoid the influence of quality of care in the first facility which may positively or negatively affect ART outcome in the first 6-months. At the second phase of data abstraction, information on each patient was obtained with respect to whether they are still alive and receiving ART services and if not, what happened?

\section{Data collection}

Data were abstracted from already existing medical records in the OSS. Each facility maintained a national patient register which captured all the variables for analysis in this study. The following data were abstracted: At T1, socio-demographics of patients, the state where OSS is located, and at T2, information on clinic follow up such as appointment keeping, drug adherence, lost to follow up, transferred out, stopped ART, death, and re-start ART. A line listing of the patients was developed on an Excel template to capture all the variables required for analysis on the study. The final Excel template merged for all the states was imported to SPSS version 20 for analysis.

\section{Ethical consideration}

Ethical clearance specifically for this study was obtained from the Nigeria Institute of Medical Research. Only information documented in routine patient care register was abstracted and use for analysis with no additional patient contacts or study specific inquiries.

\section{Study limitation}

The loss to follow up constituted most of the attrition by patients. A patient is declared lost to follow up when patient fails to show up in the clinic 90 days after the last missed appointment and all effort by the health worker to trace and return the patient to the facility has failed. Some patients assumed to be lost to follow up in this study may be alive but self-transfer to other facilities or the patient may be dead or stopped ART. Because many KPs will not provide correct contact information, it is difficult for the health worker to trace them when the need arises. This factor could result in overestimation of lost to follow up as an outcome but may also underestimate actual outcomes like death and stopped ART among the study cohort.

Similarly, the study could no way determine the status of patients who transferred to other facilities. Therefore the estimate of death, stopped ART and LTF could not be ascertained for those patients that initiated ART at the OSS but transferred to other facilities.

\section{Result}

The study was conducted between October 2016 and September 2017 in 7 OSS facilities each located in one of the 7 states supported by SHiPS for MARPS project. Data were collected at baseline (T1) and at 6-7 months (T2) after initiation of ART for each patient. A total of 3,611 KPs including their partners were initiated between October 2016 and March 2017. Out of the 3,611 KPs initiated during the period, 2351(65\%) were FSW, 688(20\%) were MSM, 339(9\%) were partners (PT) of KPs, 155(4\%) were male PWID and 78(2\%) were female PWID. The baseline characteristics and the outcomes of ART services at 6-7 months after initiation are presented below: 
Texila International Journal of Public Health

Volume 6, Issue 2, Jun 2018

Table 1. Baseline characteristic of patients

\begin{tabular}{|l|l|l|l|}
\hline Characteristics & Type & Number & Percentage \\
\hline \multirow{4}{*}{ Sex } & Male & 1135 & 31 \\
\cline { 2 - 4 } & Female & 2476 & 69 \\
\hline \multirow{5}{*}{$\begin{array}{l}\text { Occupational } \\
\text { status }\end{array}$} & None & 507 & 14.0 \\
\cline { 2 - 4 } & Primary & 720 & 19.9 \\
\cline { 2 - 4 } & Secondary & 1764 & 48.9 \\
\cline { 2 - 4 } & Tertiary & 620 & 17.2 \\
\hline Marital status & Employed & 1265 & 35.1 \\
\cline { 2 - 4 } & Student & 304 & 8.4 \\
\cline { 2 - 4 } & $\begin{array}{l}\text { Un- } \\
\text { employed }\end{array}$ & 2042 & 56.5 \\
& Married & 1185 & 33 \\
\cline { 2 - 4 } & Single & 1874 & 52 \\
\cline { 2 - 4 } & Divorced & 239 & 6.5 \\
\cline { 2 - 4 } & Separated & 26 & 0.7 \\
\cline { 2 - 4 } & Widowed & 268 & 7.3 \\
\cline { 2 - 4 } & $\begin{array}{l}\text { Co- } \\
\text { habituating }\end{array}$ & 19 & 0.5 \\
\hline Age & $\begin{array}{l}15-24 \\
\text { (young } \\
\text { person) }\end{array}$ & 933 & 25.8 \\
\cline { 2 - 4 } & $\begin{array}{l}\geq 25 \text { years } \\
\text { (adults) }\end{array}$ & 2678 & 74.2 \\
\cline { 2 - 4 } Min =15, Max=75, Mean =30.9, std = 8.7 \\
\hline
\end{tabular}

$\mathrm{N}=3611$.

Sixty-nine percent of the patients were female, while $86 \%$ had primary education and above. Unemployed accounted for $56.5 \%$ while only $35.1 \%$ were employed and the remaining $8.4 \%$ were the student. 52\% of the patients were single while $33 \%$ were married. Majority of the patients were adult (74.2\%) and the rest were the young persons (25.8\%). Mean age was 30.9 \pm 8.7 (range 15-75).

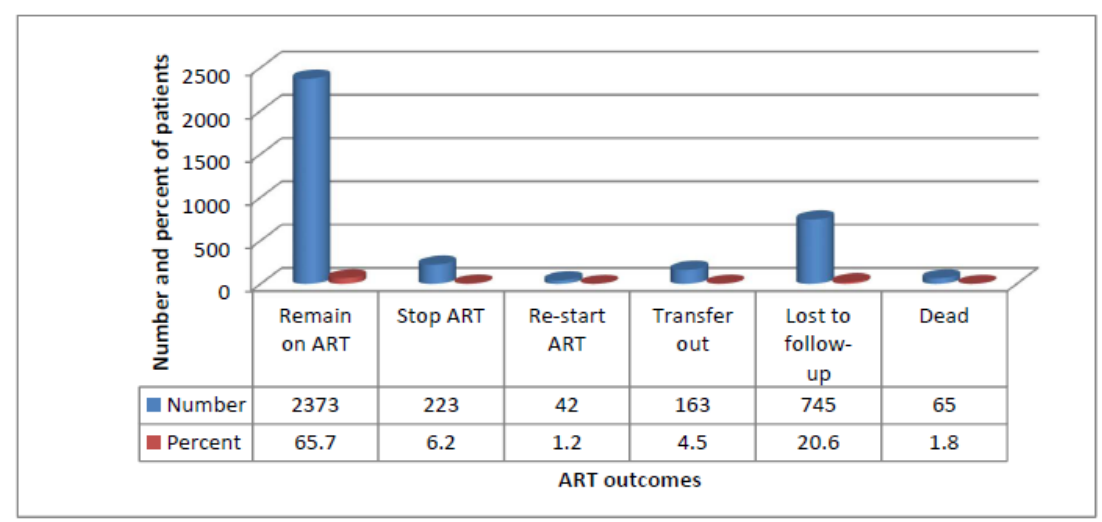

Figure 1. ART outcomes among KPs at 6-7 months after initiation on ART at OSS

Out of 3,611 initiated of ART, 65.7\% remain on ART throughout the 6-7 months. 1.2\% stopped but re-started before the end of $7^{\text {th }}$ month, $6.2 \%$ stopped ART completely, $4.5 \%$ were transferred to other facilities, while $20.6 \%$ were lost to follow up and $1.8 \%$ died. 


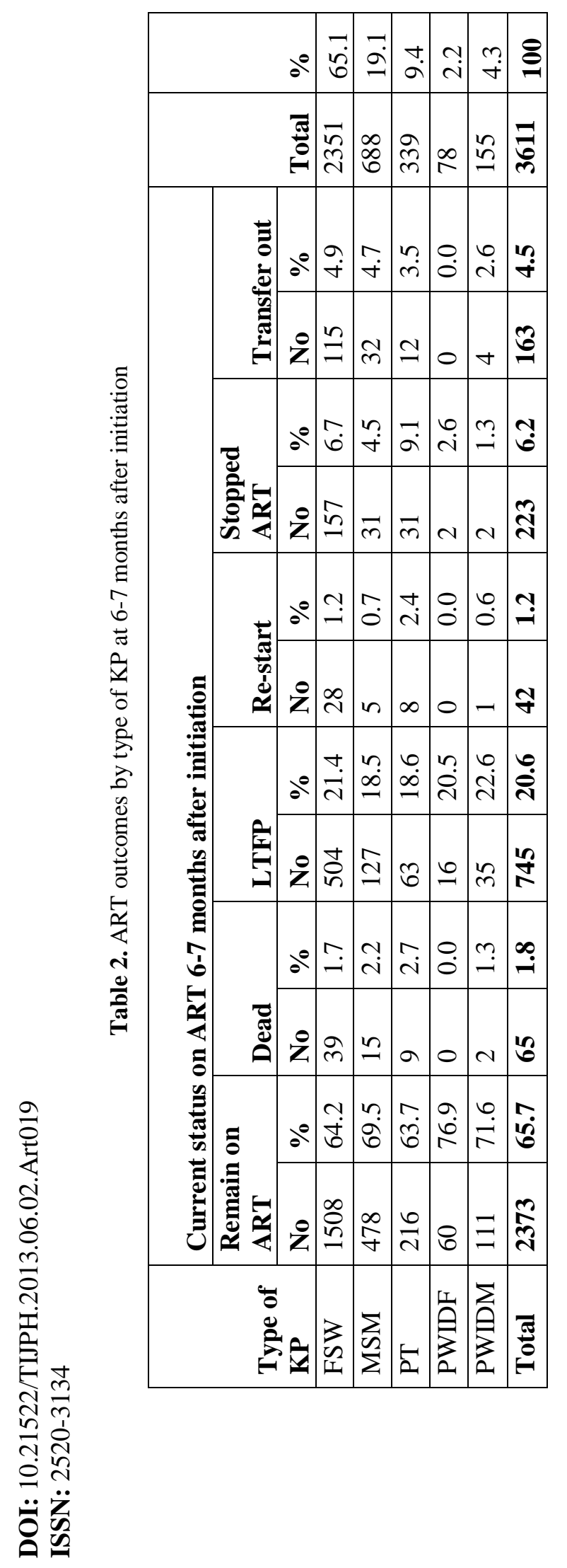


Retention rate includes those patients who remained on ART or re-start ART as at 6-7 months. PWIDF had the highest retention rate (76.9\%) followed by PWIDM (72.2\%). MSM had retention rate of $70.2 \%$, PT was $66.1 \%$ and the least was FSW with $65.4 \%$ retention rate. Most death occurred among PT $(2.7 \%)$ followed by MSM $(2.2 \%)$ then FSW (1.7\%). No death was recorded among PWIDF. PWIDM had the highest lost to followed rate with $22.6 \%$, followed by FSW (21.4\%), while PT and MSM were $18.6 \%$ and $18.5 \%$ respectively. $2.4 \%$ of PT stopped but re-started ART before the end of $7^{\text {th }}$ months followed by FSW (1.2\%). No PWIDF re-started ART. 9.1\% among PT stopped ART completely as at $7^{\text {th }}$ month followed by FSW (6.7\%) and then MSM (4.5\%). The least was among PWIDM with 1.3\%; Most transferred out occurred among FSW (4, 9\%) followed by MSM (4.7\%) and then PT (3.5\%). None of the PWIDF transferred to other facilities.

Table 3. Retention on ART at 6-7th months by age group

\begin{tabular}{|l|l|l|l|}
\hline Age group & Still on ART (\%) & Lost out of ART (\%) & Total \\
\hline $\begin{array}{l}10-24 \text { years (young } \\
\text { person) }\end{array}$ & $604(69 \%)$ & $277(31 \%)$ & 881 \\
\hline$\geq 25$ Years (old adults) & $1811(71 \%)$ & $756(29 \%)$ & 2567 \\
\hline Total & $\mathbf{2 4 1 5}$ & $\mathbf{1 0 3 3}$ & $\mathbf{3 4 4 8}$ \\
\hline
\end{tabular}

$\mathrm{X}^{2}=1.239 ; \mathrm{P}=0.27$ at $95 \% \mathrm{CI}$.

Sixty-nine percent of the young people (10-24 years) were still on ART at 6-7 months after initiation while among older people ( $\geq 25$ years) $71 \%$ were retained on ART at 6-7 months. Retention on ART at 6-7 months was not significantly different between the young people and old adults. $P$ Value $=0.27$.

Table 4. Retention on ART at 6-7 months after initiation by sex

\begin{tabular}{|l|l|l|l|}
\hline Sex & Still on ART $(\boldsymbol{\%})$ & Lost out of ART (\%) & Total \\
\hline Female & $1620(69 \%)$ & $741(31 \%)$ & 2361 \\
\hline Male & $795(73 \%)$ & $292(27 \%)$ & 1087 \\
\hline Total & $\mathbf{2 4 1 5}$ & $\mathbf{1 0 3 3}$ & $\mathbf{3 4 4 8}$ \\
\hline
\end{tabular}

$\mathrm{X} 2=7.254 ; \mathrm{P}=0.007$ at $95 \% \mathrm{CI}$, Cramer's V=0.046.

Sixty-nine percent of all females initiated on ART was still receiving ART services as at 6-7 months after initiation while $73 \%$ of all males were retained on ART at the same period. More male patients were significantly retained on ART at 6-7 months after initiation than female. $P$-Value $=$ 0.007 .

Table 5. Retention on ART at 6-7 months after initiation by educational status

\begin{tabular}{|l|l|l|l|}
\hline $\begin{array}{l}\text { Educational level } \\
\text { completed }\end{array}$ & $\begin{array}{l}\text { Still on ART } \\
(\%)\end{array}$ & Lost out of ART (\%) & Total \\
\hline Illiterate & $252(53 \%)$ & $224(47 \%)$ & 476 \\
\hline Literate & $2163(73 \%)$ & $809(27 \%)$ & 2972 \\
\hline Total & $\mathbf{2 4 1 5}$ & $\mathbf{1 0 3 3}$ & $\mathbf{3 4 4 8}$ \\
\hline
\end{tabular}

$\mathrm{X} 2=76.949 ; \mathrm{P}=0.000$ at $95 \% \mathrm{CI}$, Cramer's V $=0.149$.

Seventy-three percent of those who had at least primary education and above were retained on ART at 6-7 months, while only 53\% of those who had no education at all were retained on ART. The difference was statistically significant, $P$-Value $=0.000$. 
DOI: $10.21522 /$ TIJPH.2013.06.02.Art019

ISSN: $2520-3134$

Table 6. Retention on ART at 6-7 months by partner's status

\begin{tabular}{|l|l|l|l|}
\hline Sex partners status & Still on ART $(\boldsymbol{\%})$ & $\begin{array}{l}\text { Lost out of ART } \\
(\boldsymbol{\%})\end{array}$ & Total \\
\hline Living with partner & $768(66 \%)$ & $393(44 \%)$ & 1161 \\
\hline Living separately & $1647(72 \%)$ & $640(28 \%)$ & 2287 \\
\hline Total & $\mathbf{2 3 9 9}$ & $\mathbf{1 0 4 9}$ & $\mathbf{3 4 4 8}$ \\
\hline
\end{tabular}

$\mathrm{X} 2=12.627 ; \mathrm{P}-$ Value $=0.0001$ at $95 \% \mathrm{CI}$, Cramer's V $=0.061$.

Seventy-two percent of patients leaving separately were retained on ART while only $66 \%$ of those leaving with their partners were retained. The difference was statistically significant; P-Value $=0.001$.

Table 7. Retention on ART at 6-7months by occupational status

\begin{tabular}{|l|l|l|l|}
\hline Occupation & Still on ART (\%) & Lost out of ART (\%) & Total \\
\hline Employed & $923(77 \%)$ & $280(23 \%)$ & 1203 \\
\hline Unemployed & $1492(66 \%)$ & $753(34 \%)$ & 2245 \\
\hline Total & $\mathbf{2 4 1 5}$ & $\mathbf{1 0 3 3}$ & $\mathbf{3 4 4 8}$ \\
\hline
\end{tabular}

$\mathrm{X}^{2}=39.340$ : P-Value 0.000 at $95 \%$ CI; Cramer's V $=0.107$.

Retention on ART was highest among employed respondent $77 \%$ more than $66 \%$ among unemployed. The difference was statistically significant. P-Value $=0,000$.

\section{Discussion}

The main outcomes of the ART programme are improved quality of life and reduced mortality [26] however; patients enrolled on ART may experience other outcomes in the course of receiving treatment. Events such as retention on ART, lost to follow up, death, transfer out, re-start ART, stopped ART and measurement of CD4 and viral load are commonly used to assess the effectiveness and outcomes of the ART program. Most ART outcomes studies conducted in Sub-Saharan African reviewed long-term outcome among the general populations in the clinical settings and thus, data on short-termed outcomes particularly for KPs is lacking. Our study is a short-term assessment of ART outcomes among KPs receiving ART services in community-based One-Stop-Shops facilities. We found that $66.9 \%$ (remain on ART and re-start ART) were retained on ART, $1.8 \%$ died, $20.6 \%$ were lost to follow up, $4.5 \%$ transferred to other facilities, and $6.2 \%$ stopped ART by the end of $6^{\text {th }}$ months. This finding only shows similarity in the number of loss to follow up when compared with a recent study across 22 Sub-Saharan Africa countries [27]. The study which reviewed ART outcomes among general population after 5 years of initiation showed that $76.8 \%$ were retained on ART, $19.6 \%$ were lost to follow up and $3.5 \%$ died. Also, two similar long-termed retrospective studies conducted among the general population in Nigeria, reported similarity only on a number of retention rate and deaths but differs on the loss to follow up and stopped ART. The first study [17], reported $68 \%$ of the patients were retained on ART, with $1.8 \%$ death rate, $30.1 \%$ lost to follow up, and $0.1 \%$ stopped ART after 8 years of initiation of ART. In the second study [18], the retention rate was $61.7 \%$, the death rate was $1.1 \%$, loss to follow up was $12.3 \%$ and stopped ART was $13.9 \%$ after 4 years of initiation of ART. Even though these two studies in Nigeria were 4-8 years retrospective analysis of ART services among the general population, the retention rate in our study $(66.9 \%)$ is similar to retention rate found in the two studies $68 \%$ and $61.7 \%$ in the first and second studies respectively. Similarly, the attrition rate in our study for 6 months also collaborates with the attrition rate described by the two studies. While the first study reported that attrition was highest in the first 6 months [17], the second study reported that attrition was highest in the first 3 months [18]. It is therefore important to note that community-based ART services for KPs if well managed will have a similar outcome with ART services at clinical setting for the general population. The SHiPS for MARPS project focused on KPs and with the establishment of OSS that provided comprehensive services for KPs, this has removed the fear of stigma and discrimination which are part of reasons why KPs may be reluctant to access 
services in public health facilities. At the OSS, the staffs were trained to provide MARPS friendly services and with ambient created in the facilities, MARPS found it a convenient place even at leisure times to interact freely. Counselling was provided at every point of contact at the OSS and each patient was linked to case management officers who were constantly in communication with the patients.

Retention on ART has remained a major challenge to the success of ART services [24] particularly for KPs with social challenges that have prevented access to and utilization of health services. Patient's retention on antiretroviral therapy is required to minimize the likelihood of treatment interruptions and discontinuation [28], which not only reduces patient morbidity and mortality but also minimizes the risk of developing drug resistance and promote viral suppression [29]. Despite these and other benefits, retention on ART is Sub-Saharan African even among the general population remains low at the various point due to high attrition rate. In our study, PWID had highest retention rate $(74.6 \%)$ more than MSM (70.2\%). This is contrary to the finding in a study in the US [20] that MSM had better linkage and retention on ART more than other KPs. However, a study in Vietnam has shown retention rate among PWID as high as $88.4 \%$ after 6 months on ART [22]. FSW had the least retention rate $(65.4 \%)$ but highest transfer out rate (4.9\%) than MSM (4.7\%). Both brothel base and non-brothel based FSWs are highly mobile and can change location from time to time. Because of multiple client patronages for FSWs who are normally found in a location, an HIV positive FSW is more likely to relocate for fear of her status being disclosed to potential customers by her competing colleagues. Thus, a good number of FSW declared loss to follow up may be a case of self-transfer because of relocation to other places.

There are several definitions of loss to follow up on ART [30] which vary across different programs and studies [31] but in this study, a patient is declared loss to follow up if 90 days after the last missed clinic appointment, all efforts to trace and return the patient to service has failed [27]. Lost to follow-up is a major challenge that hinders the success of antiretroviral treatment [32] and it is the major cause of attrition from ART services. In sub-Saharan African countries, rates of loss to follow up vary as exhibited in the two studies in Nigeria and the study in 22 Sub-Saharan African countries. In another systemic review of patient retention following ART initiation in Sub-Saharan African countries, it was evident that 1 year after initiation, approximately $25 \%$ of patients were no longer in cares, with loss to follow up figures escalating to $40 \%$ after 2 years [32]. However, it is reasonable to think that a patient lost to follow up may belong to any of the following categories: death, change of residence, self-transfer to other facilities without the knowledge of the treatment facility. An operational research study in Northern Malawi [33] to determine the true outcomes of patients declared as loss to follow up has shown that, $50 \%$ had died, $15 \%$ had stopped therapy, and $8 \%$ had transferred to another clinic and failed to inform their original clinic of this move. From field experience, some KPs will not provide accurate contact information thus making it impossible to trace them when the need arises. A study in Gabon found that part of the reason why patients do not adhere to care is that of poor supportive counselling by healthcare providers [34]. On SHiPS for MARPS project, adherence counselling was important and it was provided at various levels starting from point of testing. When the patient is positive and enrolled in care, all point of services at the OSS provided supportive counselling; starting with the adherence counsellor, the clinician, the pharmacist and the case management officer who is attached to the patient.

While death may be due to the complication of the disease, it is unclear why patients on ART would decide to stop their medication despite the known fact that antiretroviral drugs are life-saving drugs. Further study is therefore required to trace and ascertain reasons why some patients decide to stop taking their drugs. In Nigeria, some traditional healers and churches have laid claim to cure of HIV/AIDS and some of these patients who deliberately stopped ART have been traced to such areas. In our study, $1.2 \%$ of the patients stopped ART and re-start again. From our experience, some of the patients may stop because they wanted to try an alternative treatment, but when their symptoms resurface or get worsened, they return to ART services.

Various factors [16] [34] [35] [36], have been found to impact negatively on patient retention including; fear of stigma and social exclusion, distance and lack of access to facility, lack of money for transportation to the clinic, younger age, lack of education and minimal social support for the 
patients, the burden of multiple vulnerabilities, policy involving risk behaviours and mental health, work and child care burden, self transfer to more convenient clinic without the knowledge of health provider, death, suboptimal quality of health care due to practitioners' work overload and medical record keeping problems. In this study, even though retention among the adult ( $\geq 25$ years) was $71 \%$ more than $69 \%$ among young people (15-24 years), the difference was not statistically significant. $\mathrm{P}=$ 0.27 , at $95 \%$ CI. However, a study in Zimbabwe reported that younger adults suffered more attrition from ART care compared to older adults [37] Perhaps, the number of young persons who participated in this study $(30 \%)$ is not sufficient to show a significant difference. We also found that illiterate patients were less likely to be retained on ART. P $=0.000$ at $95 \% \mathrm{CI}$. This finding collaborate with other studies in Nigeria which reported that patients with post-secondary education $(\mathrm{HR}=0.80$, 95\%CI: $0.65-0.99)$ or other unspecified education ( $\mathrm{HR}=0.61,95 \% \mathrm{CI}$ : $0.41-0.93)$ were less likely to become lost to follow up compared to individuals with no primary or some primary education [38] and that lower-than-secondary level education is a predictor of loss to follow up [18]. Since years of education can be a measure of health literacy, lower education levels may lead to lower retention of ART. Furthermore, a patient with lower education level may have difficulties in comprehending the instructions at ART clinics [39]. This finding suggests that providing educational materials in local languages may contribute to improved retention [18]. This study also found that being a male was associated with higher retention rate on ART P $=0.007$ at $95 \%$ CI. However, this is contrary to other findings where attrition was higher among male patients [18], [40] due to poorer clinical status prior to initiating ART [41]. We also found that patients who were employed were retained on ART more than unemployed or being a student, $\mathrm{P}=0.000$ at $95 \% \mathrm{CI}$. An earlier study [39] had described that those who were unemployed had a higher rate of treatment discontinuation than employed patients. This may be attributed to financial constraint which is one of the reasons why some patients are unable to access health services [34]. Moreover, unemployment may be considered as a factor negatively affecting the quality of life and it has been shown that lower quality of life is associated with lower retention [39]. Another finding in our study was that patients who were single or live separately from their sex partners had a higher retention rate $\mathrm{P}=0.001$ at $95 \% \mathrm{CI}$. This is contrary to another finding [39] that being single were associated with loss to follow up.

\section{Conclusion}

Community-based ART services among key populations when properly managed will show similar outcomes as in the general population in the clinical settings.

The recent WHO guideline recommended "test and treat" which will facilitate the attainment of UNAIDS 90-90-90 goals. While implementation of this guideline will increase the coverage of ART among KPs, the major challenge will be how to link and retain identified positives on ART. With the high level of stigmatization of people living with HIV/AIDS, KPs face an additional barrier in accessing health services and even when they do; maintaining them on the service could still be a challenge. Most studies on retention rate were conducted on long-term analysis of general population. Currently, there is no baseline data describing retention rate in a KP focused ART services in Nigeria. This study has provided short-term baseline data on retention rate and other ART outcomes in a KP focused services.

A loss to follow up was the major cause of attrition on ART in this study but comparable to loss to follow described among the general population. While some of the patients declared loss to follow up maybe dead or self-transfer to other facilities, others may have stopped ART or receiving some nonspecific treatment. The grave danger associated with stopping antiretroviral drugs is the development of drug resistance which will mar the efforts already made to control the epidemic. Further study is therefore required to trace and ascertain reasons why some patients decide to stop taking their drugs.

The finding from this study suggests that achieving UNAIDS 90.90.90 goals is possible in Nigeria if only KPs focused intervention could be innovative early enough to identify causes of loss to follow up and address them on time. Factors predicting improved retention rates are: being single, higher education, being a male, and gainfully employed. 


\section{Acknowledgement}

The authors gratefully acknowledge the patients and the incredible work and dedication of the OSS staffs and particularly the MIS staff and the state M\&E officers who ensure that accurate data was abstracted for this study. None of the authors have any conflict of interest to declare.

\section{References}

[1].Abebe Megerso, ${ }^{1}$ Sileshi Garoma, ${ }^{1}$ Tolosa Eticha, ${ }^{2}$ Tilaye Workineh, ${ }^{1}$ Shallo Daba, ${ }^{3}$ Mihretu Tarekegn, ${ }^{3}$ andZelalem Habtamu3 (2016); Predictors of loss to follow-up in antiretroviral treatment for adult patients in the Oromia region, Ethiopia HIV AIDS (Auckl). 2016; 8: 83-92. doi: 10.2147/HIV.S98137.

[2].Andreas D Haas, Elizabeth Zaniewski, Nanina Anderegg, Nathan Ford, Matthew P Fox et al (2018) Retention and mortality on antiretroviral therapy in sub-Saharan Africa: collaborative analyses of HIV treatment programmes. Journal of ineternational AIDS society February 2018 https://doi.org/10.1002/jia2.25084.

[3].Annals of Internal Medicine (2012); Guidelines for Improving Entry Into and Retention in Care and Antiretroviral Adherence for Persons with HIV: Evidence-Based Recommendations from an International Association of Physicians in AIDS Care Panel. http://annals.org/aim/fullarticle/1170890.

[4].Anthony D Harries, 1, 2 Rony Zachariah, 3 Stephen D Lawn, 2, 4 and Sydney Rosen5, 6 (2010) Strategies to improve patient retention on antiretroviral therapy in sub-Saharan Africa Trop Med Int Health. 2010 Jun; 15(s1): 70-75. doi: 10.1111/j.1365-3156.2010.02506.x.

[5].Avert (2016); Global HIV and AIDS statistics. https://www.avert.org/global-hiv-and-aids-statistics.

[6].Avert (2016); HIV/AIDS in Nigeria; Available at https://www.AVERT.org/Professionals/HIV-aroundworld/Sub-Saharan-Africa/Nigeria.

[7].Averts (2018); HIV and AIDS in Nigeria. Available at https://www.avert.org/professionals/hiv-aroundworld/sub-saharan-africa/nigeria.

[8].Azadeh Khalatbari Limaki1, Behnam Farhoudi1, Mehrnaz Rasoolinejad2, Saeed Safari3 Patient Retention in Antiretroviral Therapy Programs; A Retrospective Cohort Study in a Triangular HIV Clinic. DOI: 10.22086/GMJ.V6I2.819 http://gmj.ir/index.php/gmj/article/view/819/html.

[9].Bryan E. Shepherd, Meridith Blevins, Lara M. E. Vaz, Troy D. Moon, Aaron M. Et al (2013); Impact of Definitions of Loss to Follow-up on Estimates of Retention, Disease Progression, and Mortality: Application to an HIV Program in Mozambique. American Journal of Epidemiology, Volume 178, Issue 5, 1 September 2013, Pages 819-828, https://doi.org/10.1093/aje/kwt030.

[10]. C.M.J. Matyang, K.C. Takarinda, P.Owiti, T. Utasa-Apollo, O. Mugurungi et al (2016); Outcomes of antiretroviral therapy among younger versus older adolescents and adults in an urban clinic, Zimbabwe. Public Health Action 2016 Jun 21; 6(2):97-104. Doi: 10.5588/pha.15.0077.

[11]. CA Onoka,1 BS Uzochukwu,2 O E Onwujekwe,3 C Chukwuka,4 J Ilozumba,5 et al. (2012); Retention and loss to follow-up in antiretroviral treatment programmes in Southeast Nigeria. Pathog Glob Health. 2012 Mar; 106(1): 46-54.doi: 10.1179/2047773211Y.0000000018

https://www.ncbi.nlm.nih.gov/pmc/articles/PMC4001511.

[12]. Cameron D. Factors associated with retention in HIV care at Sediba Hope Medical Centre. S Afr J HIV Med. 2015; 16 (1), Art. \#347, 6 pages. http://dx.doi. org/10.4102/sajhivmed.v16i1.347.

[13]. Charurat M, Oyegunle M, Benjamin R, Habib A, Eze E, Ele P, et al. (2010) Patient Retention and Adherence to Antiretrovirals in a Large Antiretroviral Therapy Program in Nigeria: A Longitudinal Analysis for Risk Factors. PLoS ONE 5(5): e10584. https://doi.org/10.1371/journal.pone.0010584.

[14]. Dalhatu I1, Onotu D1, Odafe S1, Abiri O2, Debem H1, Agolory S3, (2016) Outcomes of Nigeria's HIV/AIDS Treatment Program for Patients Initiated on Antiretroviral Treatment between 2004-2012. PLoS One. 2016 Nov 9; 11(11):e0165528. doi: 10.1371/journal.pone.0165528. Collection 2016.

[15]. doi: 10.5144/0256-4947.2015.303;

[16]. Elvin H. G. Denis N. Andrew K. Yao Z. Paula B. et al (2011); Retention in Care among HIV-Infected Patients in Resource-Limited Settings: Emerging Insights and New Directions. Available at Curr HIV/AIDS Rep https://www.ncbi.nlm.nih.gov/pmc/articles/PMC3021803/.

[17]. Elvin H. Geng, David R. Bangsberg, Nicolas Musinguzi, Nneka Emenyonu, Mwebesa Bosco Bwana, et al (2010); Understanding Reasons for and Outcomes of Patients Lost to Follow-Up in Antiretroviral Therapy Programs in Africa Through a Sampling-Based Approach J Acquir Immune Defic Syndr. 2010 Mar; 53(3): 405411. doi: 10.1097/QAI.0b013e3181b843f0. 
DOI: $10.21522 /$ TIJPH.2013.06.02.Art019

ISSN: $2520-3134$

[18]. Federal ministry of health, Nigeria (2014); integrated biological and behavioural surveillance survey (IBBSS) 2014.

Available

at

https://www.google.com.ng/search?q=11.+Federal+ministry+of+health\%2C+Nigeria+(2014)\%3B+Integrated+b iological+and+behavioural+surveillance+survey\&oq $=11 .+$ Federal+ministry+of+health $\% 2 \mathrm{C}+$ Nigeria+(2014)\%3 B+Integrated+biological+and+behavioural+surveillance+survey\&aqs=chrome..69i57.1253j0j7\&sourceid=chro me\&ie $=$ UTF- 8 .

[19]. Federal ministry of health, Nigeria (2014); National HIV sero-prevalence sentinel survey among pregnant women attending antenatal clinic in Nigeria. Available at

https://www.google.com.ng/search?q=12.+Federal+ministry+of+health\%2C+Nigeria+(2014)\%3B+National+HI V+sero-

prevalence+sentinel+survey+among+pregnant+women+attending+antenatal+clinic+in+Nigeria\&oq=12.+Federa 1+ministry+of+health\%2C+Nigeria+(2014)\%3B+National+HIV+sero-

prevalence+sentinel+survey+among+pregnant+women+attending+antenatal+clinic+in+Nigeria\&aqs=chrome.. 6 9i57.1456j0j7\&sourceid=chrome\&ie=UTF- 8 .

[20]. Health care worker attitudes and behaviours towards key populations in South Africa and Zambia: a crosscountry analysis within the HPTN 071 (PopART) trial AIDS 2016 Conference, Durban, South Africa available at http://programme.aids2016.org/Abstract/Abstract/4352.

[21]. HIV cascade framework for key population 2015. Available at https://www.fhi360.org/sites/default/files/media/documents/linkages-hiv-cascade-framework-oct15.pdf

[22]. HIV clinical resource (2017); Benefit and risk of antiretroviral therapy. Available at https://www.hivguidelines.org/adult-hiv/antiretroviral-therapy/goals-benefits-risks/.

[23]. HIV.gov 2016, the Global HIV/AIDS Epidemic. https:/www.hiv.gov/hiv-basics/overview/data-andtrends/global-statistics.

[24]. http://www.who.int/mediacentre/news/releases/2015/hiv-treat-all-recommendation/en/.

[25]. International AIDS Society (2014); Maximizing the benefits of antiretroviral therapy for key populations. Available at http://www.iasociety.org/WebContent/File/KAPs_WhitePaper_2014.pdf.

[26]. Janssen S, Wieten RW, Stolp S, Cremers AL, Rossatanga EG, Klipstein-Grobusch K, et al. (2015) Factors Associated with Retention to Care in an HIV Clinic in Gabon, Central Africa. PLoS ONE 10(10): e0140746. https://doi.org/10.1371/journal.pone.0140746.

[27]. Jennifer L. Weinberg and Carrie L. Kovarik, MD (2010); The WHO Clinical Staging System for HIV/AIDS, American Medical Association Journal Ethics, Volume 12, Number 3: 202-206.

[28]. Katerina AC, Moupali D, and Grant NC (2011); Linkage and Retention in HIV Care among Men Who Have Sex with Men in the United States. Available at

http://cid.oxfordjournals.org/content/52/suppl_2/S214.full.

[29]. Kudakwashe C. Takarindaab, Anthony D. Harriesbc Ray W. Shiraishid Tsitsi Mutasa-Apolloa Abu AbdulQuaderd Owen Mugurungia (2015); Gender-related differences in outcomes and attrition on antiretroviral treatment among an HIV- infected patient cohort in Zimbabwe: 2007-2010. International Journal of Infectious Diseases. Volume 30, January 2015, Pages 98-105 https://doi.org/10.1016/j.ijid.2014.11.009.

[30]. Mazvita Naome Mberi, Lazarus Rugare Kuonza, Nomathemba Michelle Dube, Cornelius Nattey, Samuel Manda and, Robert Summers (2015); Determinant of loss to follow in patients on antiretroviral treatment, South Africa, 2004-2012: a cohort study. BMC Health Services Research201515:259. https://doi.org/10.1186/s12913015-0912-2.

[31]. MEASURE Evaluation; Key Populations; Available at https://www.measureevaluation.org/our-work/keypopulations.

[32]. Musa BM, Coker M, Bussell S, Aliyu M, Babashani M, Muhammad H et al. (2015) Long-term outcomes of antiretroviral therapy in an adult HIV program: a 10-year retrospective cohort study in Kano, Nigeria. Ann Saudi Med 2015; 35(4): 303-311.

[33]. N MlangeniI; F SenkubugeII (2016); Antiretroviral therapy programme outcomes in Tshwane district, South Africa: A 5-year retrospective study. SAMJ, S. Afr. med. j. vol.106 n.4 Cape Town Apr. 2016 http://dx.doi.org/10.7196/samj.2016.v106i4.9375.

[34]. Nguyen DB, Do NT, Shiraishi RW, Le YN, Tran QH, Huu Nguyen H, et al. (2013) Outcomes of Antiretroviral Therapy in Vietnam: Results from a National Evaluation. PLoS ONE 8(2): e55750. https://doi.org/10.1371/journal.pone.0055750. 
[35]. PAHO; Towards a Caribbean Consensus. Improving Access to Key Populations to Comprehensive HIV Services available

http://www.paho.org/hq/index.php?option=com_docman\&task=doc_view\&gid=23834\&Itemid=270\&lang=en. [36]. Priya Lall, Sin How Lim, Norliana Khairuddin, Adeeba Kamarulzaman (2015); Review: An urgent need for research on factors impacting adherence to and retention in care among HIV-positive youth and adolescents from key populations. Journal of international AIDS society. https://doi.org/10.7448/IAS.18.2.19393.

[37]. Rose Wilcher (2017); Call for paper: Optionizing the impact of key population programming across HIV cascade. Available at https://researchforevidence.fhi360.org/call-for-papers-optimizing-the-impact-of-keypopulation-programming-across-the-hiv-cascade.

[38]. S. Krishnaratne1, H. Mathema2, A. Stang13, G. Hoddinott4, V. Bond5, J. Hargreaves1;

[39]. Seema T. Meloni, Charlotte A. Chang, Geoffrey Eisen, Toyin Jolayemi, Bolanle Banigbe, Prosper I. Okonkwo, Phyllis J. Kanki (2016) Long-Term Outcomes on Antiretroviral Therapy in a Large Scale-Up Program in Nigeria. PLOS https://doi.org/10.1371/journal.pone.0164030;

[40]. Van der Kop ML, Ekström AM, Awiti-Ujiji O, Chung MH, Mahal D, et al. (2014) Factors Associated with Attrition from HIV Care during the First Year after Antiretroviral Therapy Initiation in Kenya. J AIDS Clin Res 5:354. doi:10.4172/2155-6113.1000354.

[41]. WHO (2014); Consolidated guidelines on HIV prevention, diagnosis, treatment and care for key populations. Available at

http://apps.who.int/iris/bitstream/10665/128048/1/9789241507431_eng.pdf?ua=1\&ua=

[42]. WHO (2015); Treat all people living with HIV, offer antiretrovirals as additional prevention choice for people at "substantial" risk; News release ! GENEVA. Available at.

[43]. WHO (2016); Consolidated Guidelines on HIV Prevention, Diagnosis, Treatment and Care for Key Populations - 2016 Update. https://www.ncbi.nlm.nih.gov/books/NBK379696/.

[44]. Yibeltal A, Achamyeleh A, Meskele L, Lut L, Edwin W, and Wim V (2014); Scaling up antiretroviral treatment and improving patient retention in care: lessons from Ethiopia, 2005-2013 Globalization and Health201410:43 DOI: 10.1186/1744-8603-10-43. 